Alexandre de Lima Spinola

\title{
Modelagem e Controle Não Linear da Direção de um Veículo Terrestre
}

\author{
Dissertação de Mestrado
}

Dissertação apresentada como requisito parcial para obtenção do título de Mestre pelo Programa de PósGraduação em Engenharia Elétrica da PUC-Rio.

Orientadores: Marcos Azevedo da Silveira Mauro Speranza Neto

Rio de Janeiro, 19 de dezembro de 2003 
Alexandre de Lima Spinola

\title{
Modelagem e Controle Não Linear da Direção de um Veículo Terrestre
}

Dissertação apresentada como requisito parcial para obtenção do título de Mestre pelo Programa de PósGraduação em Engenharia Elétrica da PUC-Rio. Aprovada pela Comissão Examinadora abaixo assinada.

\author{
Marcos Azevedo da Silveira \\ Orientador \\ DEE/PUC-Rio \\ Mauro Speranza Neto \\ Co-Orientador \\ DEM/PUC-Rio \\ Fernando Ribeiro da Silva \\ DEMM/IME \\ Carlos Térsio Correa da Silva \\ DEE/PUC-Rio
}

Ney Augusto Dumont Coordenador Setorial do Centro Técnico Científico - PUC-Rio

Rio de Janeiro, 19 de dezembro de 2003 
Todos os direitos reservados. É proibida a reprodução total ou parcial do trabalho sem autorização da universidade, do autor e do orientador.

\section{Alexandre de Lima Spinola}

Engenheiro de Controle e Automação formado pela PUCRio em 2001, ingressou para o Mestrado, no Departamento de Engenharia Elétrica da PUC-Rio em agosto de 2001, na área de Processamento de Sinais e Controle de Processos. Enquanto aluno de graduação estagiou na indústria offshore, na DSND Consub, no desenvolvimento de um robô submarino de operação remota nacional. Trabalhou ainda com criação de aplicativos dedicados para análise estrutural mecânica, na EasyCAE Designer. Após se formar, trabalhou nos laboratórios do DEE, como Auxiliar de Ensino e Pesquisa e como Estagiário em Docência, este último como requisito obrigatório por ser bolsista da CAPES. Já no final do Mestrado voltou a trabalhar na indústria offshore, como engenheiro de sistemas de controle submarinos, na FMC CBV Subsea, projetando, especificando e provendo manutenção a sistemas de controle multiplexados para auxílio à extração de petróleo no fundo do mar. Ao longo do curso de mestrado criou, junto com mais quatro sócios, a BioGénie Projetos, empresa atuante na área biomédica de produção de próteses em geral, projetando equipamentos para o mercado odontológico além de patentes de métodos e aplicações. Dentro da BioGénie é o responsável pela área de pesquisa e desenvolvimento de novas tecnologias e sua aplicação direta aos novos produtos. 
Ficha Catalográfica

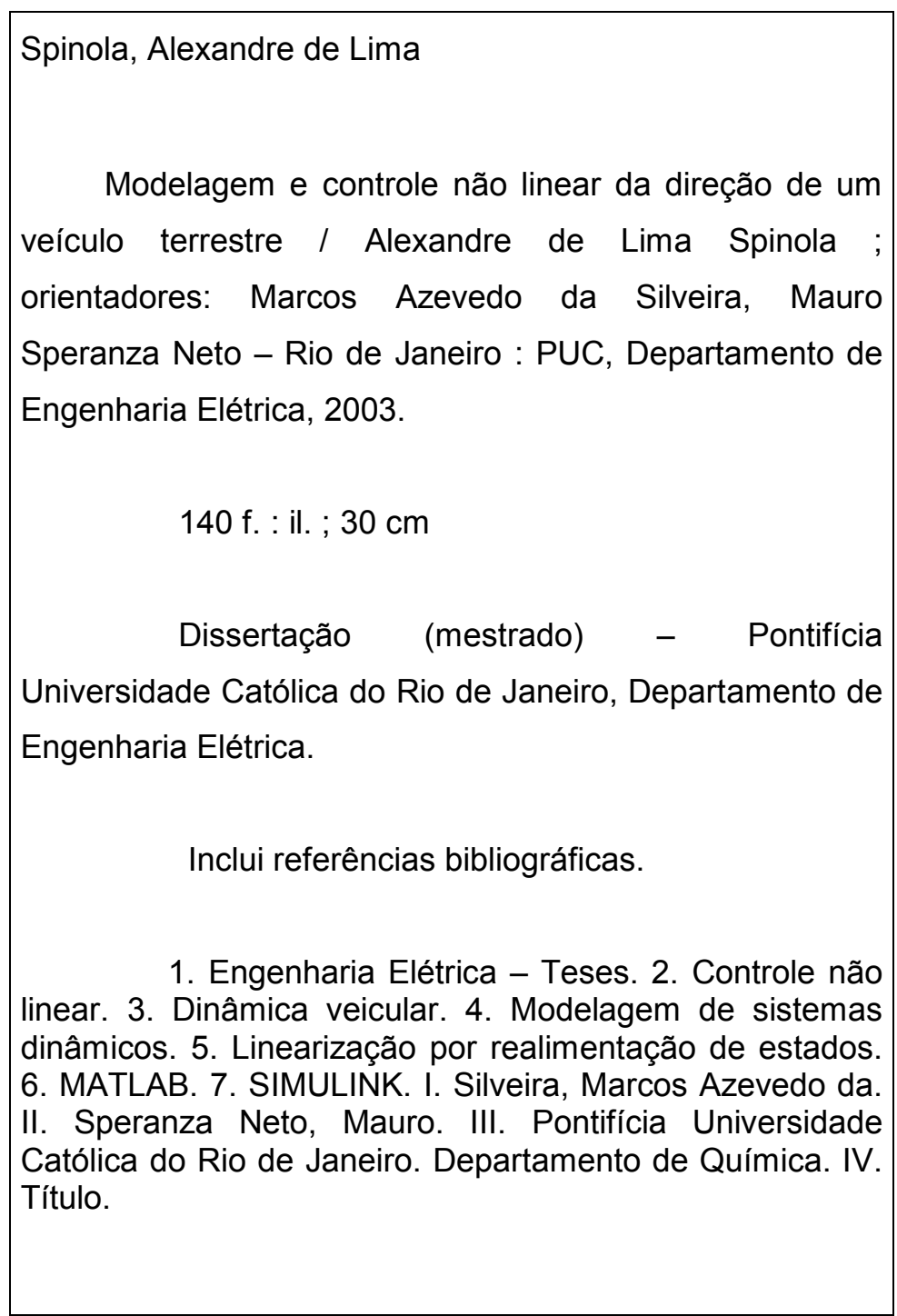


"Só conheço uma liberdade, e essa é a liberdade do pensamento".

Antoine de Saint-Exupéry

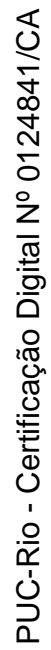




\section{Agradecimentos}

Aos meus orientadores Professores Marcos Azevedo da Silveira e Mauro Speranza Neto, pelo estímulo e parceria para a realização deste trabalho e, acima de tudo, pela amizade.

A CAPES e a PUC-Rio, pelos auxílios concedidos, sem os quais este trabalho não existiria.

Aos meus pais Oscar e Lúcia, pela educação, incentivo e amor de sempre.

À minha irmã Cláudia, por sua alegria contagiante e pelas conversas de irmãos.

Aos meus amigos de longa data, por todo o apoio, paciência e compreensão nas horas de sumiço total.

Aos professores Fernando e Térsio, que participaram da Comissão examinadora.

A todos os professores e funcionários dos departamentos de Engenharia Elétrica e de Engenharia Mecânica, pelos ensinamentos e pela ajuda.

A todos os amigos e familiares que de uma forma ou de outra me estimularam ou me ajudaram a seguir em frente. 


\section{Resumo}

Spinola, Alexandre de Lima. Modelagem e Controle Não Linear da Direção de um Veículo Terrestre. Rio de Janeiro, 2003. 140p. Dissertação de Mestrado - Departamento de Engenharia Elétrica, Pontifícia Universidade Católica do Rio de Janeiro.

Modelagem e Controle Não Linear da Direção de um Veículo Terrestre descreve um estudo em dinâmica veicular no qual inicialmente apresenta-se um modelo analítico para representar a geração de forças longitudinais e laterais no contato do pneu com o solo. Em seguida é desenvolvido, para um automóvel de passeio, um modelo não linear de 4 graus de liberdade (velocidades longitudinal, lateral, de guinada e de rolagem), e a sua linearização. Expande-se esse modelo para um de 8 graus de liberdade, no qual inclui-se o movimento de rotação axial de cada uma das quatro rodas, e consideram-se os movimentos do veículo somente no plano, sem efeitos de pitch ou bounce, mas apresentando alguma relação de distribuição de cargas devido ao roll. Todos os modelos em malha aberta são validados através de simulações computacionais em diversas condições típicas de operação. Na segunda parte desse trabalho é apresentada a estratégia proposta para o tratamento do problema de controle direcional do veículo em uma manobra qualquer, empregando a metodologia da linearização por realimentação, tendo como base o modelo linear de 4 graus de liberdade. São analisados os resultados encontrados através de simulação computacional para a malha fechada com diferentes combinações de parâmetros, empregando os modelos não lineares de 4 e 8 graus de liberdade. Conclui-se discutindo a possibilidade de generalização deste procedimento para diferentes aplicações em Dinâmica Veicular.

\section{Palavras-chave}

1. Controle Não Linear; 2. Dinâmica Veicular; 3. Modelagem de Sistemas Dinâmicos; 4. Linearização por Realimentação de Estados; 5. Matlab; 6. Simulink 


\section{Abstract}

Spinola, Alexandre de Lima. Modeling and Non Linear Control of a Ground Vehicle's Steering. Rio de Janeiro, 2003. 140p. Msc. Dissertation Electric Engineering Department, Pontifical Catholic University of Rio de Janeiro.

Modeling and Non Linear Control of a Ground Vehicle's Steering describes a study in vehicle dynamics, which presents an analytic model representing the generation of longitudinal and lateral forces at the contact patch between tire and ground. Next it is developed, for a typical passenger car, a non-linear model with four degrees of freedom (longitudinal, lateral, yaw and roll velocities), and its linearization. This model is then expanded to another one with eight degrees of freedom, which includes the axial rotation of each one of the four wheels, and considers the vehicle's movement only at a known plane, without pitch and bounce effects, but including some load distribution among the wheels, due to roll. Computational simulations in various typical operation conditions validate all open loop models. The second part of this work presents the proposed strategy for directional control of a vehicle at any type of manoeuvre, using the feedback linearization methodology, directly applied to the linear four degrees of freedom model. The results obtained trhough computational simulation for a closed loop model with different parameters are analysed using both nonlinear four and eight degrees of freedom models. The possibility of generalizing this procedure to distinct applications in Vehicle Dynamics is, then, discussed.

\section{Keywords}

1. Non Linear Control; 2. Vehicle Dynamics; 3. Modeling of Dynamic Systems; 4. Feedback Linearization 5. Matlab; 6. Simulink 


\section{Sumário}

1 Introdução

2 Modelagem do Pneu

2.1. Introdução

2.2. Forças e momentos dos pneus

2.3. Resistência ao rolamento

2.4. Força de tração ou frenagem e deriva longitudinal (slip e skid) 24

2.5. Força lateral e ângulo de deriva 37

2.6. Caracterização do comportamento de pneus em curvas 38

2.7. Teoria da elipse de aderência 42

2.8. Simulação do modelo do pneu 48

3 Modelo de Quatro Graus de Liberdade $\quad 50$

3.1. Construção do modelo não linear 50

3.1.1. Modelagem do pneu 55

3.1.2. Simplificando o modelo não linear para a dinâmica lateral 61

3.2. Simulação do modelo de quatro graus de liberdade 68

4 Modelo de Oito Graus de Liberdade $\quad 74$

4.1. Construção do modelo não linear 74

4.2. Simulação do modelo de oito graus de liberdade 86

5 Controle por Linearização da Realimentação de Estados 97

5.1. Teoria de controle 97

5.1.1. Linearização no sentido entrada-saída 97

5.1.2. Formas normais 99

5.1.3. Análise dos estados não observáveis 103

5.1.4. Estabilidade assintótica local 105

6 Controle Aplicado ao Modelo de Quatro Graus de Liberdade 106 
6.1. Formas normais 108

6.2. Análise dos estados não observáveis 112

6.3. Análise de estabilidade 114

6.4. Controle para rastreamento de trajetória 115

6.5. Simulação do modelo com quatro graus de liberdade 116

7 Conclusões e Trabalhos Futuros 122

8 Referências Bibliográficas 125

Anexo 1 Tentativa de Controle do Modelo de Oito Graus de Liberdade 128 $\begin{array}{ll}\text { A1.1 Linearização da função de entrada } & 128\end{array}$

A1.2 Controle aplicado ao modelo de oito graus de liberdade 130

A1.3 Formas normais 133

A1.4 Análise de estabilidade em regime estacionário 135

Anexo 2 Lista de Variáveis e Dados Numéricos 138 


\section{Lista de figuras}

Figura 1 - Representação de um modelo de dinâmica veicular 19

$\begin{array}{ll}\text { Figura } 2 \text { - Sistema de eixos do pneu } & 20\end{array}$

Figura 3 - Efeito de onda estacionária 23

Figura 4 - Características de pneus $\quad 24$

Figura 5 - Comportamento do pneu durante a tração (aceleração positiva) 26

Figura 6 - Variação do esforço de tração com a deriva longitudinal 27

Figura 7 - Comportamento do pneu sob frenagem (desaceleração) 35

Figura 8 - Comportamento do pneu sujeito a uma força lateral 37

Figura 9 - Curva de força lateral para os dois tipos de pneu 38

Figura 10 - Conceito de elipse de aderência 41

Figura 11 - Construção da elipse de aderência 43

Figura 12 - Curvas de forças laterais sob ação de força normal de 4000N 48

Figura 13 - Curvas de forças longitudinais sob ação de força normal de 4000N 49

Figura 14 - Elipse de aderência de um pneu a 4000N de força normal 49

Figura 15 - Determinando as acelerações em x e y 51

Figura 16 - Diagrama de corpo livre para equilíbrio de torques 53

Figura 17 - Identificação dos ângulos de deriva 55

Figura 18 - Orientação de componentes das forças nos pneus $\quad 60$

Figura 19 - Geometria de Ackermann 65

Figura 20 - Modelo de 4 graus de liberdade em ambiente de simulação $\quad 68$

Figura 21 - Função de entrada degrau $\quad 69$

Figura 22 - Resposta ao degrau: ângulo de guinada $\quad 70$

$\begin{array}{ll}\text { Figura } 23 \text { - Resposta da velocidade lateral ao degrau } & 70\end{array}$

Figura 24 - Deslocamento no referencial global do modelo de 4 graus de $\begin{array}{ll}\text { liberdade } & 71\end{array}$

Figura 25 - Ângulo de esterçamento constante para movimento em curva 71

Figura 26 - Deslocamento lateral do modelo de 4 graus ao longo do tempo 72

Figura 27 - Deslocamento do modelo no plano XY, a uma curvatura constante 72

Figura 28 - Variação do ângulo de guinada ao longo do tempo 73

Figura 29 - Equilíbrio de forças $\quad 76$ 
Figura 30 - Diagrama de forças nas rodas $\quad 79$

Figura 31 - Curvas de torque do motor $\quad 81$

Figura 32 - Modelo de 8 graus de liberdade em ambiente de simulação $\quad 87$

Figura 33 - Função de entrada para o modelo de 8 graus de liberdade 88

Figura 34 - Ângulo de guinada para mudança de pista 89

Figura 35 - Deslocamento do modelo de 8 graus de liberdade para a mudança de pista $\quad 89$

Figura 36 - Visão geral do deslocamento no referencial global 90

Figura 37 - Forças laterais durante o movimento de mudança de faixa 91

Figura 38 - Deriva longitudinal durante o movimento de mudança de faixa 91

Figura 39 - Forças normais na mudança de faixa 92

Figura 40 - Compensação de velocidades nas rodas durante a mudança de faixa 92

Figura 41 - Teste de estabilidade do modelo de oito graus de liberdade em curva

Figura 42 - Deslocamento do modelo 8GL em curva de raio constante

Figura 43 - Ângulos de guinada e rolagem do modelo de 8 graus de liberdade 95

Figura 44 - Forças normais durante o movimento de curva de raio constante 95

Figura 45 - Teste de estabilidade em regime permanente 114

Figura 46 - Diagrama da estratégia de controle 115

Figura 47 - Diagrama de blocos no ambiente de simulação do modelo de 4 graus

Figura 48 - Controlador com pólos próximos à origem 117

Figura 49 - Melhoria do sistema após deslocar pólos para a esquerda 118

Figura 50 - Rastreamento da trajetória desejada 118

Figura 51 - Acompanhamento de uma curva de raio constante 119

Figura 52 - Velocidade lateral durante realização de curva de raio constante 120

Figura 53 - Aplicação de nos estados do modelo de 4 graus $-\mathrm{SNR}=-3 \mathrm{~dB} \quad 121$

Figura 54 - Aplicação de ruído no sinal de controle - SNR = -4dB 121

Figura 55 - Quebra da estabilidade da dinâmica interna 136 